\title{
Barbed reposition pharyngoplasty in multilevel robotic surgery for obstructive sleep apnoea
}

\section{La barbed reposition pharyngoplasty nella chirurgia multilivello robotica per il trattamento delle apnee ostruttive in sonno}

\author{
C. VICINI' ${ }^{1}$, G. MECCARIELLO ${ }^{1}$, G. CAMMAROTO ${ }^{2}$, M. RASHWAN $^{3}$, F. MONTEVECCHI ${ }^{1}$
}

${ }^{1}$ Department of Head-Neck Surgery, Otolaryngology, Head-Neck and Oral Surgery Unit, Morgagni Pierantoni Hospital, Forlì, Italy; ${ }^{2}$ Department of Otolaryngology, University of Messina, Italy; ${ }^{3}$ Department of Otolaryngology,

Suez Canal University Teaching Hospitals, Ismailia, Egypt

\section{SUMMARY}

The surgical treatment of obstructive sleep apnoea in patients who are non-compliant with continuous positive airway pressure therapy still represents a valid alternative. In recent years, the multilevel approach is becoming more diffuse in routine surgical practice, especially since the introduction of transoral robotic surgery. Barbed reposition pharyngoplasty in multilevel robotic surgery for OSA may represent a valid option to surgically approach the soft palate. Herein, we describe the technique and preliminary results of our experience.

KEY WORDS: Obstructive Sleep Apnoea Syndrome $\bullet$ Surgery $\bullet$ Medical failure $\bullet$ Procedure $\bullet$ Robotic

\section{RIASSUNTO}

Il trattamento chirurgico delle apnee ostruttive in sonno rappresenta una valida alternativa per i pazienti non complianti al ventilatore CPAP. Negli ultimi anni, l'approccio chirurgico multilivello sta divenendo pratica comune, soprattutto dall'introduzione della chirurgia robotica. La Barbed Reposition Pharyngoplasty nella chirurgia robotica multilivello per OSA potrebbe rappresentare una valida opzione per il trattamento del palato molle. In questo lavoro descriviamo la tecnica e risultati preliminari della nostra esperienza.

PAROLE CHIAVE: Sindrome di apnea ostruttiva del sonno $\bullet$ Chirurgia $\bullet$ Fallimento medico $\bullet$ Procedure $\bullet$ Chirurgia robotica

\section{Introduction}

The surgical treatment of obstructive sleep apnoea (OSA) in patients who are non-compliant with continuous positive airway pressure (CPAP) therapy is still a valid alternative. In recent years, the multilevel approach is becoming more diffuse in routine surgical practice, especially since the introduction of transoral robotic surgery (TORS) ${ }^{1}$. Although in some patients the main site of nocturnal obstruction is the base of tongue (BOT), the soft palate collapse is commonly associated. In the past 2 decades, several surgical techniques have been introduced to manage soft palate collapse ${ }^{2}$. Recently, our group introduced a new surgical approach to soft palate in OSA patients that is promising in terms of efficacy, feasibility and teachability ${ }^{3}$. Herein, we describe the technique in a multilevel robotic surgery setting.

\section{Materials and methods}

The study was conducted in compliance with our Institutional Review Board requirements. Between January
2014 and December 2015 at Otolaryngology, Head-Neck and Oral Surgery Unit, Department of Head Neck Surgery, Morgagni Pierantoni Hospital, Azienda USL Romagna, 85 TORS for OSA were carried out. The barbed reposition pharyngoplasty (BRP) was associated in 43 cases. We included all patients who completed a one-year post-operative full overnight sleep study.

All procedures are performed with the patient under general anesthesia who signed an informed consent. Robotic surgery is usually the first procedure to be performed as previously described ${ }^{4}$. Tonsillectomy may be performed robotically or in a traditional approach depending on the surgeon's choice. An important factor is meticulous sparing of the palatoglossus (PGM) and palatopharyngeus (PPM) muscles and as much as possible the mucosal covering both pillars. The centre point closest to the posterior nasal spine (PNS) was marked at the palatal spine, and the pterygomandibular raphe (PMR) in both sides were located by digital palpation and marked (Fig. 1). Next, a partial incision was carried out using a pinpoint bowie monopolar scalpel at the inferior (caudal) part of the PPM. 


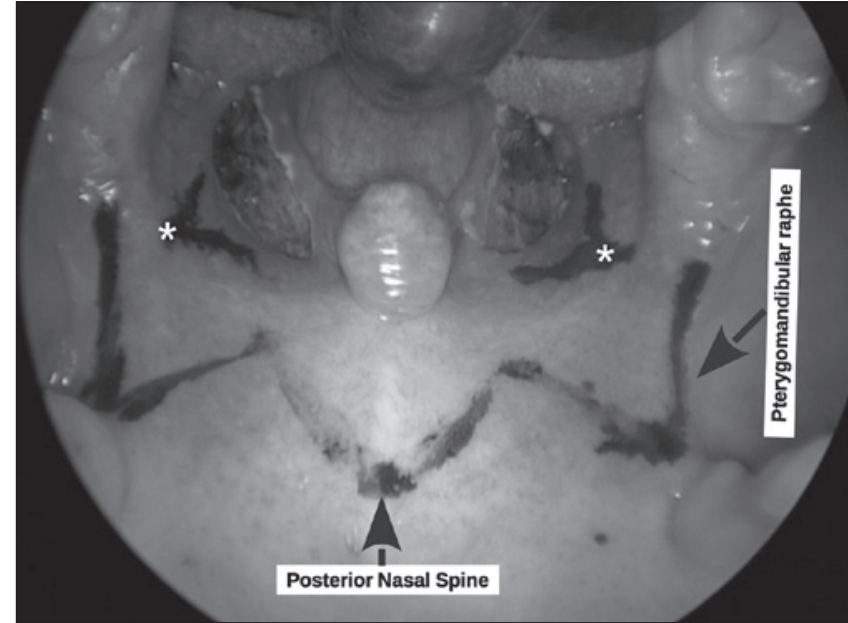

Fig. 1. Marking the landmarks and drawing the suture line including the triangles of superolateral corner of the tonsillar fossa on both sides $\left({ }^{*}\right)$.

A full thickness triangle was removed at the superolateral corner of the tonsillar fossa to obtain a wider and squared oropharyngeal inlet (Fig. 2). We usually use two needles polydioxanone (PDO) barbed suture (size $036 \times 36 \mathrm{~cm}$, taper 1/2 circle QUILL Knotless Tissue Closure Device, Surgical Specialities Corp., Vancouver, CA). One needle is introduced at the centre point and then passed laterally within the muscular layer of the soft palate (point 1 to 2 in Fig. 3); the thread is then pulled until it hangs at the central transition zone, which is a free zone between the two directions of the thread. After passing through point 2, the needle is inserted within PMR at the most superior part of the raphe at one side (point 3 ). The needle again is reintroduced close to point of exit, passing around the PMR, until it exits into the tonsillectomy bed, then through the upper part of the PPM sparing the overlaying mucosa (point 4). The posterior pillar is entered at the junction between the upper third and the lower two-thirds. Then, the

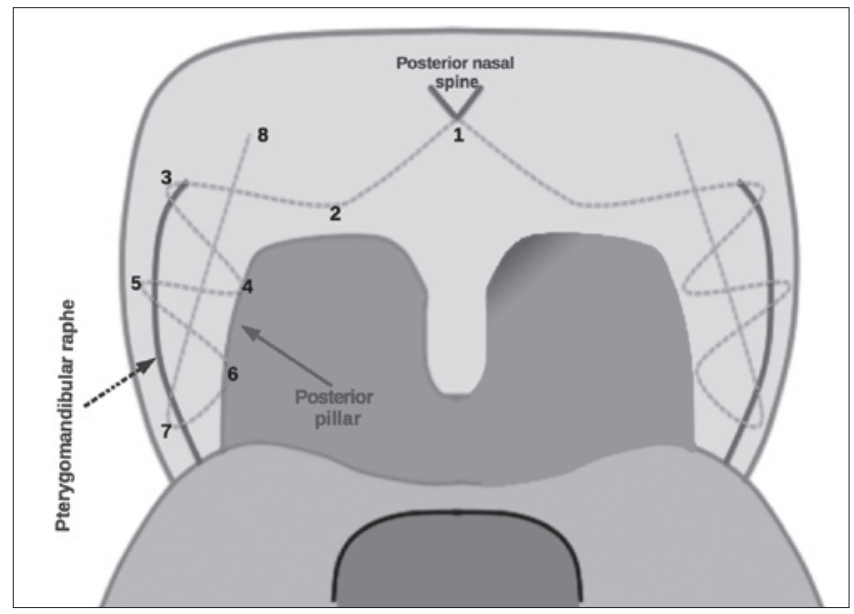

Fig. 3. Scheme of suture points.

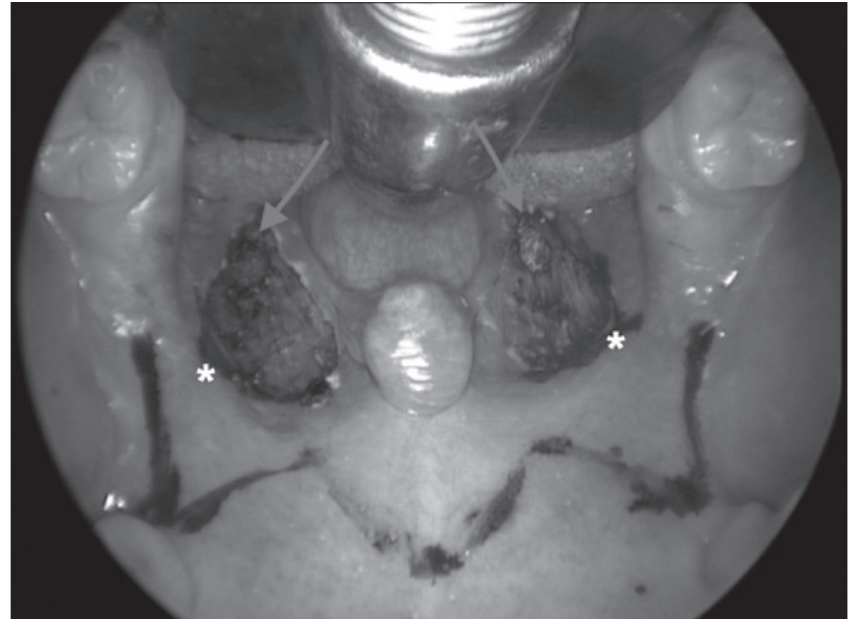

Fig. 2. Incision and releasing of the palatopharyngeal muscle(arrows). Full thickness triangles were removed at the superolateral corner of the tonsillar fossa on both sides $\left(^{*}\right)$.

needle is again passed back through the tonsillectomy bed and then this suture will be suspended around the PMR (point 5); gentle traction is then applied on the thread only and no knots are made. This leads to stable repositioning of the posterior pillar to more lateral and anterior location without any knot, and then this stitch is repeated at least three times between raphe and muscle till the lower pole of the muscle is reached (point 5 to 7 ). The opposite side is done by the same way. Finally, each thread comes out at the PMR of the same side, to lock the stitches and prevent looseness; a superficial stitch in the opposite direction is taken, and then the thread is cut while pushing the tissue downward for more traction (point 8). The closure of pillars by single stitches is a surgeon's preference. The final result is widening of the oropharyngeal lateral wall and forward sustaining of the soft palate (Fig. 4). The multilevel surgery is concluded, if required, with a nasal procedure.

Statistical analysis was performed with STATA 12.0 software (Stata Corp., College Station, TX, USA).

\section{Results}

Currently, only 10 of 43 patients who underwent BRP associated with TORS completed the follow-up with a full overnight sleep study after one-year post-operatively. The preoperative and post-operative characteristics of patients are shown in Table 1. The median age was 64 years (range 45-74), and the median preoperative apnoeahpyopnoea index (AHI) and body mass index (BMI) were 32.7 (10-58) and 27.9 (21.7-32.5), respectively. The median preoperative Epworth Sleepiness Scale (ESS) score was 12 (7-15). The usual collapse pattern highlighted by drug-induced sleep endoscopy (DISE) was complete antero-posterior oropharyngeal collapse (O4ap) with involvement of the epiglottis $(\mathrm{L}+)$. The median post-opera- 

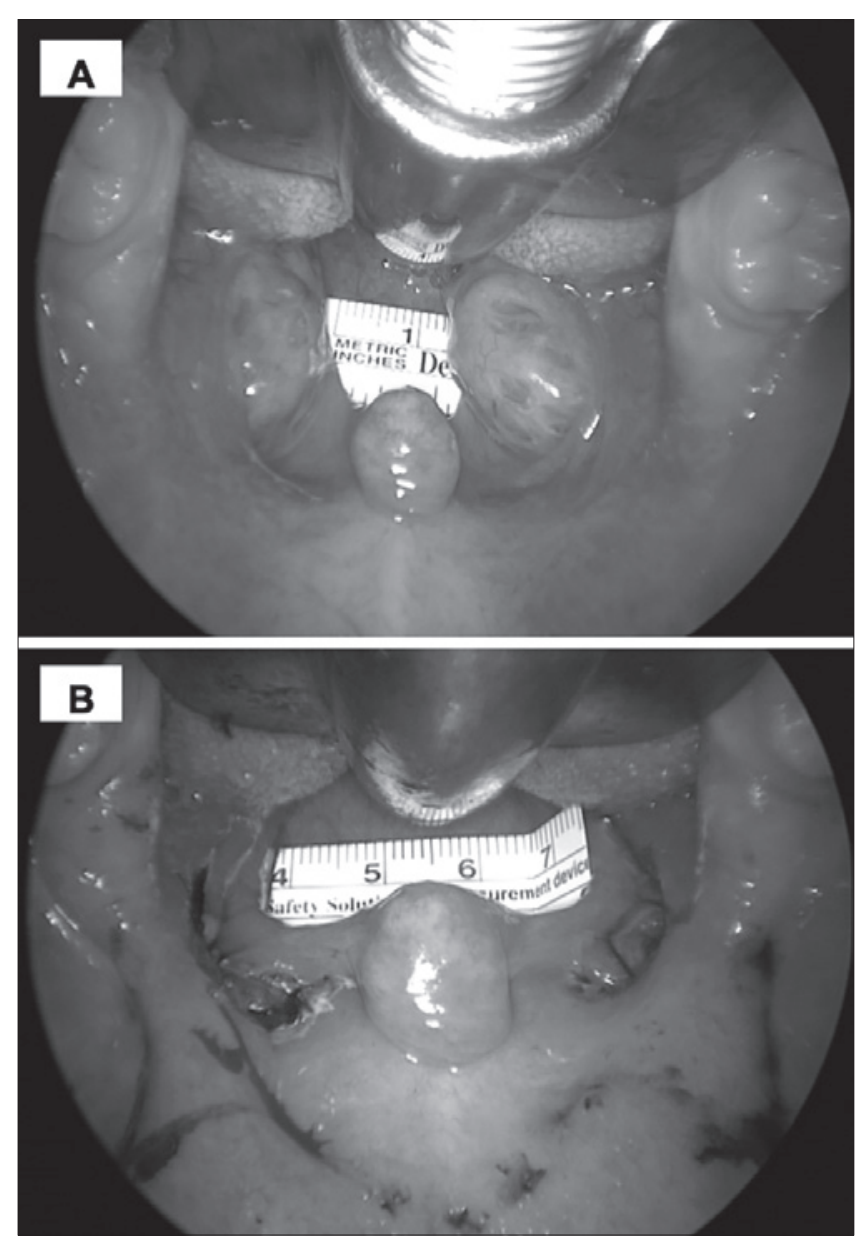

Fig. 4. A) The starting view before the tonsillectomy and the barbed pharyngoplasty. B) Immediate post-operative endoscopic view. Note the increased space between soft palate and posterior pharyngeal wall.

tive values of AHI, BMI and ESS were 16.9 (6.1-45), 28.9 (21.7-31.4) and $4(0-12)$ respectively. The mean TORS time was $44.8 \pm 22.3$ minutes, whilst the mean pharyngo- plasty time was $13.5 \pm 1.5$ minutes. The overall procedure time was $79.3 \pm 36$ minutes whether nose surgery and/or tracheostomy were done.

The treatment was effective in 7 of 10 patients. Patients \#2, \#3 and \#6 experienced no improvements after surgical treatment. Although further investigation will be required to assess the failure, it is important to note that their BMI increased at the post-operative evaluation and these patients had a severe OSA syndrome. No patients experienced intra-operative, post-operative or delayed complications. Patients \#5 and \#6 experienced a transient dysphagia that spontaneously resolved within one month.

\section{Discussion}

Since 2003, various surgical techniques have been published to address the obstruction caused by the soft palate and lateral pharyngeal wall ${ }^{2}$. However, uvulopalatopharyngoplasty (UPPP) is still the most widely used despite its low success rate and long-term, post-surgical side effects. In 2014, Salamanca et al. ${ }^{5}$ described a novel and compelling technique using barbed sutures in anterior pharyngoplasty. The use of this kind of suture showed interesting results in simple snorers or mild OSA-patients with significant fewer complications and reduced operative time. Based on this report, our group developed a new variant of ESP using the barbed sutures ${ }^{3}$. This preliminary study shows promising results in reducing the operative time, efficacy on OSA and a very simple learning curve. Post-operative pain is a common problem in palatal surgery and some patients experience an unacceptable level of intense post-operative pain ${ }^{6-8}$. In BRP, the healing time and post-operative pain is related mostly to tonsillectomy; in fact, in patients who underwent previously tonsillectomy, usually very minimal post-operative pain and rapid oral feeding is reported in our sleep surgery experience. Neither velopharyngeal insuffi-

Table I. Patient characteristics.

\begin{tabular}{|c|c|c|c|c|c|}
\hline Patient & Pre-op BMI & $\begin{array}{c}\text { Pre-op } \\
\text { AHI }\end{array}$ & $\begin{array}{c}\text { Pre-op } \\
\text { DISE } \\
\text { classification }^{\dagger}\end{array}$ & Post-op BMI & $\begin{array}{c}\text { Post-op } \\
\text { AHI }\end{array}$ \\
\hline$\# 1$ & 26.7 & 42.5 & N304apH4apL- & 26.7 & 19.4 \\
\hline \#2 & 29.4 & 20.1 & N204apH3L+ & 31.4 & 33.8 \\
\hline \#3 & 25.7 & 43 & N304apH4apL+ & 29.8 & 44 \\
\hline \#4 & 29.6 & 34.6 & N304cH4apL+ & 29.6 & 24 \\
\hline$\# 5$ & 21.7 & 30.7 & N003apH4apL- & 21.7 & 14.4 \\
\hline$\# 6$ & 29 & 58 & N004apH4apL+ & 29.2 & 45 \\
\hline \#7 & 32.5 & 35.7 & N303cH3apL+ & 28.6 & 11.8 \\
\hline \#8 & 22.6 & 26 & N304apH4apL+ & 22.4 & 11 \\
\hline$\# 9$ & 22.6 & 16.6 & N003tH4CL+ & 22.6 & 6.1 \\
\hline$\# 10$ & 30 & 10 & N302cH1tL- & 29.3 & 7.5 \\
\hline
\end{tabular}

Pre-op = pre-operative; Post-op = post-operative; $B M I$ = body mass index; $A H I$ = apnea-hypopnea index; DISE = drug-induced sleep endoscopy

${ }^{+}$according to Vicini et al. The nose oropharynx hypopharynx and larynx (NOHL) classification: a new system of diagnostic standardised examination for OSAHS patients. Eur Arch Otorhinolaryngol 2012;269:1297-300. 
ciency nor nasopharyngeal regurgitation are noted. In this report, we describe our early experience using this technique in multilevel robotic surgery. The technique fits well within the robotic surgery framework because its inherent ease and rapid execution allows reducing surgical times that have a considerable impact on costs.

\section{Conclusions}

Barbed reposition pharyngoplasty in multilevel robotic surgery for OSA may represent a valid option to surgically approach the soft palate. Further investigations are needed to evaluate the effectiveness in a large population.

\section{References}

1 Vicini C, Montevecchi F, Pang K, et al. Combined transoral robotic tongue base surgery and palate surgery in obstructive sleep apnea-hypopnea syndrome: expansion sphincter pharyngoplasty versus uvulopalatopharyngoplasty. Head Neck 2014;36:77-83.

2 Carrasco-Llatas M, Marcano-Acuña M, et al. Surgical re- sults of different palate techniques to treat oropharyngeal collapse. Eur Arch Otorhinolaryngol 2015;272:2535-40.

3 Vicini C, Hendawy E, Campanini A, et al. Barbed reposition pharyngoplasty (BRP) for OSAHS: a feasibility, safety, efficacy and teachability pilot study. "We are on the giant's shoulders". Eur Arch Otorhinolaryngol 2015;272:30653070 .

4 Vicini C, Montevecchi F, Magnuson JS. Robotic surgery for obstructive sleep apnea. Curr Otorhinolaryngol Rep 2013;1:130-6.

5 Salamanca F, Costantini F, Mantovani M, et al. Barbed anterior pharyngoplasty: an evolution of anterior palatoplasty. Acta Otorhinolaryngol Ital 2014;34:434-8.

6 Virtaniemi J, Kokki H, Nikanne E, et al. Ketoprofen and fentanyl for pain after uvulopalatopharyngoplasty and tonsillectomy. Laryngoscope 1999;109:1950-4.

7 Kokki H, Nikanne E, Aho M, et al. Pain intensity after laseruvulopalatoplasty and tonsillectomy. Otolaryngol Head Neck Surg 2003;128:273-9.

8 Ismail SA, Mowafi HA. Preoperative peritonsillar lornoxicam infiltration is not superior to intravenous lornoxicam for pain relieffollowing tonsillectomy in adults. Eur J Anaesthesiol 2010;27:807-11. 